\title{
Stability Analysis of Numerical Methods for a 1.5-Layer Shallow-Water Ocean Model
}

\author{
Guang-an Zou, ${ }^{1,2}$ Bo Wang, ${ }^{3}$ and $\mathrm{Mu} \mathrm{Mu}{ }^{1}$ \\ ${ }^{1}$ Key Laboratory of Ocean Circulation and Wave, Institute of Oceanology, Chinese Academy of Sciences, Qingdao 266071, China \\ ${ }^{2}$ University of Chinese Academy of Sciences, Beijing 100049, China \\ ${ }^{3}$ Institute of Applied Mathematics, Henan University, Kaifeng 475004, China \\ Correspondence should be addressed to Mu Mu; mumu@qdio.ac.cn
}

Received 7 March 2013; Accepted 12 September 2013

Academic Editor: Jong Hae Kim

Copyright (C) 2013 Guang-an Zou et al. This is an open access article distributed under the Creative Commons Attribution License, which permits unrestricted use, distribution, and reproduction in any medium, provided the original work is properly cited.

A 1.5-layer reduced-gravity shallow-water ocean model in spherical coordinates is described and discretized in a staggered grid (standard Arakawa C-grid) with the forward-time central-space (FTCS) method and the Leap-frog finite difference scheme. The discrete Fourier analysis method combined with the Gershgorin circle theorem is used to study the stability of these two finite difference numerical models. A series of necessary conditions of selection criteria for the time-space step sizes and model parameters are obtained. It is showed that these stability conditions are more accurate than the Courant-Friedrichs-Lewy (CFL) condition and other two criterions (Blumberg and Mellor, 1987; Casulli, 1990, 1992). Numerical experiments are proposed to test our stability results, and numerical model that is designed is also used to simulate the ocean current.

\section{Introduction}

The shallow-water model is a set of partial differential equations (PDEs), which derived from the principles of conservation of mass and conservation of momentum (the NavierStokes equations). Because the horizontal length scale is much greater than the vertical length scale, under this condition, the conservation of mass implies that the vertical velocity of the fluid is very small. It can be shown from the momentum equations that horizontal pressure gradients are due to the displacement of the pressure surface (or free surface) in a fluid, and that vertical pressure gradients are nearly hydrostatic [1]. The vertical integrating allows the vertical velocity to be removed from the equations; this is a classical derivation of the shallow-water system.

The situations in fluid dynamics where the horizontal length scale is much greater than the vertical length scale are very common; that is to say, the vertical acceleration of the fluid can be negligible. The flow of water over a free surface is a ubiquitous physical phenomenon that has aroused many scientists and engineers' interest. For instance, if we consider the Coriolis forces in shallow-water model (the Coriolis term exists because we describe flows in a reference frame fixed on earth), this set of equations is particularly well suited for the study and numerical simulations of a large class of geophysical phenomena, such as atmospheric flows, ocean circulation, coastal flows, tides, tsunamis, and river and lake flows [2-10].

The shallow-water equations are derived from the NavierStokes equations that are nonlinear partial differential equations, which describe the motion of fluids. The nonlinearity makes most problems difficult or impossible to solve and it is the main contributor to the turbulence. Mathematicians and physicists believe that the turbulence can be found through an understanding of solutions to the Navier-Stokes equations. However, in the mathematical field, mathematicians have not yet proven that in three dimensions solutions always exist (existence), or that if they do exist, then they do not contain any singularity (that is smoothness). These are called the Navier-Stokes existence and smoothness problems; this is one of the seven most important open problems (the Millennium Prize Problems) in mathematics [11]. Therefore, it is also a challenge to make substantial progress toward the exact solution of shallow-water equations. 
Research on numerical methods for the solution of the shallow-water system has attracted much attention; numerical simulation is an effective tool in solving them and a great variety of numerical methods have been developed to solve this system [12-22]. The numerical models which are based on the shallow-water equations, especially for finite difference numerical models, have been successfully applied to study the ocean circulation; for example, the low-frequency variability and bifurcation structure of wind-driven ocean circulation [5-7], the shallow-water model for the study of the Gulf Stream and its extension region [23-25], the Kuroshio current and its extension system [26-30], and so on. Although few people discuss the stability of numerical models, only several papers give the stability conditions of the simple formulation and linearized shallow-water equations [20, 3136]. In this paper, we use the discrete Fourier analysis method and the Gerschgorin circle theorem to study the stability of the shallow-water numerical models and give a series of necessary conditions for the selection criteria of time step size.

The remainder of the paper is organized as follows. In Section 2, the brief description of 1.5-layer reduced-gravity shallow-water ocean model has been introduced. In Section 3, we use the FTCS method and the Leap-frog finite difference scheme to solve the shallow-water equations in a staggered grid. In Section 4, the discrete Fourier method combined with the Gerschgorin circle theorem is used to analyze the stability of these two numerical methods. In Section 5, numerical examples are given to test our results. The conclusions are given in Section 6.

\section{Matematical Model}

The mathematical derivation of the shallow-water equations can be found in many fluid dynamics books [37] and has already been presented by many authors. In our study, 1.5layer shallow-water equations in spherical coordinates are nondimensionalized using the length scale $r_{0}$, which is radius of the earth, the mean depth of the upper layer $H$, a characteristic horizontal velocity scale $U$, a time scale $r_{0} / U$, and a wind-stress scale $\tau_{0}$ (see $[27,38]$ ).

The nondimensional equations are governed by the following reduced-gravity, nonlinear partial differential equations:

$$
\begin{gathered}
\varepsilon\left(\frac{\partial u}{\partial t}+\frac{u}{\cos \theta} \frac{\partial u}{\partial \phi}+v \frac{\partial u}{\partial \theta}-u v \tan \theta\right)-v \sin \theta \\
=\frac{-\varepsilon F}{\cos \theta} \frac{\partial h}{\partial \phi}+E\left(\nabla^{2} u-\frac{u}{\cos ^{2} \theta}-\frac{2 \sin \theta}{\cos ^{2} \theta} \frac{\partial v}{\partial \phi}\right)+F_{\phi} \\
\varepsilon\left(\frac{\partial v}{\partial t}+\frac{u}{\cos \theta} \frac{\partial v}{\partial \phi}+v \frac{\partial v}{\partial \theta}+u^{2} \tan \theta\right)+u \sin \theta \\
=-\varepsilon F \frac{\partial h}{\partial \theta}+E\left(\nabla^{2} v-\frac{v}{\cos ^{2} \theta}+\frac{2 \sin \theta}{\cos ^{2} \theta} \frac{\partial u}{\partial \phi}\right)+F_{\theta} \\
\frac{\partial h}{\partial t}+\frac{1}{\cos \theta}\left[\frac{\partial(h u)}{\partial \phi}+\frac{\partial(h v \cos \theta)}{\partial \theta}\right]=0
\end{gathered}
$$

in which $u$ is the zonal velocity, $v$ is the meridional velocity, $\phi$ is the coordinate in the zonal direction, $\theta$ is the coordinate in

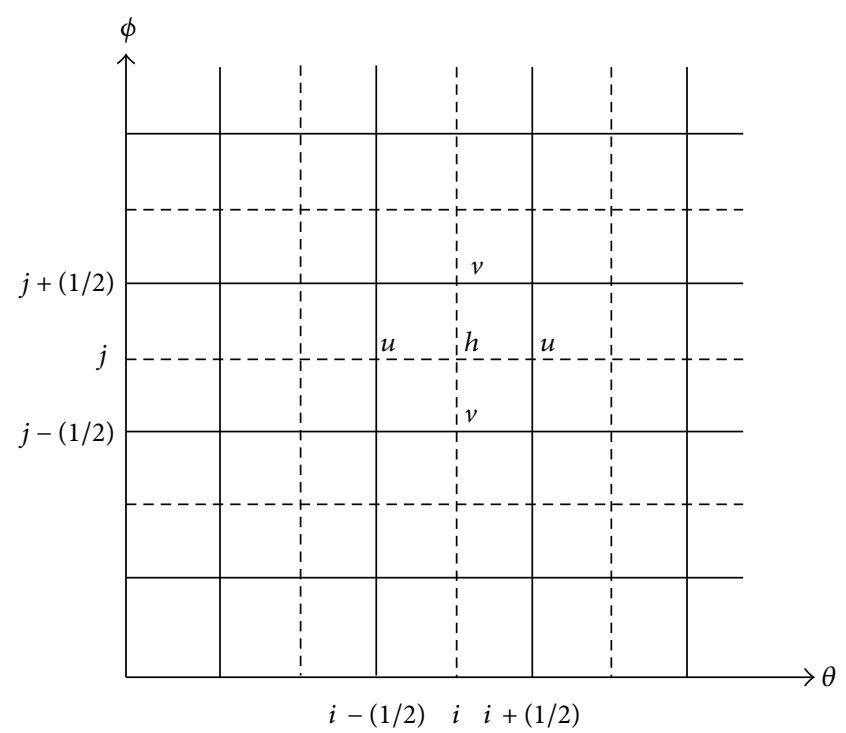

FIGURE 1: Staggered grid on space domain.

the meridional direction, and $h$ is the thickness of the upper ocean. The parameters in the equations are $\varepsilon=U / 2 \Omega r_{0}, E=$ $A_{H} / 2 \Omega r_{0}^{2}$, and $F=g^{\prime} H / U^{2}$, where $\Omega$ is the rotation rate of the earth, $A_{H}$ is the lateral friction coefficient, and $g^{\prime}$ is the reduced gravity.

The terms $F_{\phi}$ and $F_{\theta}$ in (1)-(2) are defined as follows:

$$
F_{\phi}=\frac{\alpha \tau_{\phi}}{h}-\mu u, \quad F_{\theta}=\frac{\alpha \tau_{\theta}}{h}-\mu \nu,
$$

where $\alpha=\tau_{0}(2 \Omega \rho H U)$ is wind stress coefficient ( $\tau_{0}$ is the amplitude of wind stress) and $\tau_{\phi}$ and $\tau_{\theta}$ are the zonal component and meridional component of wind stress, respectively. In addition, $\mu=\gamma /(2 \Omega)$, where $\gamma$ is the interfacial friction coefficient.

\section{Finite Difference Schemes}

If one discretizes the domain to a grid with equally spaced points with a spacing of $\Delta \phi$ in the $\phi$-direction, $\Delta \theta$ in the $\theta$-direction, and $\Delta t$ in the $t$-direction, we define $u_{i, j}^{n}=$ $u\left(\theta_{i}, \phi_{j}, t_{n}\right)$ with $\theta_{i}=\theta_{0}+i \Delta \theta, \phi_{j}=\phi_{0}+j \Delta \phi$, and $t_{n}=n \Delta t$ for $i=0,1, \ldots, M, j=0,1 \ldots, N$, and $n=1,2, \ldots, Q$, where $M, N$, and $Q$ are positive integers. The variables $u, v$, and $h$ are evaluated at a staggered grid (standard Arakawa C-grid) as shown in Figure 1; then the shallow-water equations can be solved by using finite difference method.

3.1. The FTCS Method. The forward difference approximation is used for the time derivative and the central difference approximation for the spatial derivatives. The difference approximation of (1)-(3) is given by

$$
\begin{gathered}
\frac{u_{i+1 / 2, j}^{n+1}-u_{i+1 / 2, j}^{n}}{\Delta t}+\frac{u^{*}\left(u_{i+1 / 2, j+1}^{n}-u_{i+1 / 2, j-1}^{n}\right)}{2 \Delta \phi} \\
+\frac{v^{*}\left(u_{i+3 / 2, j}^{n}-u_{i-1 / 2, j}^{n}\right)}{2 \Delta \theta}-f^{*} v_{i, j+1 / 2}^{n}
\end{gathered}
$$




$$
\begin{gathered}
=-\frac{F^{*}\left(h_{i, j+1}^{n}-h_{i, j-1}^{n}\right)}{2 \Delta \phi} \\
+E^{*}\left(\frac{u_{i+1 / 2, j+1}^{n}-2 u_{i+1 / 2, j}^{n}+u_{i+1 / 2, j-1}^{n}}{\Delta \phi^{2}}\right. \\
\left.+\frac{u_{i+3 / 2, j}^{n}-2 u_{i+1 / 2, j}^{n}+u_{i-1 / 2, j}^{n}}{\Delta \theta^{2}}\right) \\
\frac{v_{i, j+1 / 2}^{n+1}-v_{i, j+1 / 2}^{n}+\frac{u^{*}\left(v_{i, j+3 / 2}^{n}-v_{i, j-1 / 2}^{n}\right)}{2 \Delta \phi}}{\Delta t}+H^{*} \tau_{\phi}-\Phi u_{i+1 / 2, j}^{n}, \\
+\frac{v^{*}\left(v_{i+1, j+1 / 2}^{n}-v_{i-1, j+1 / 2}^{n}\right)}{2 \Delta \theta}+f^{*} u_{i+1 / 2, j}^{n} \\
=-\frac{F\left(h_{i+1, j}^{n}-h_{i-1, j}^{n}\right)}{2 \Delta \theta} \\
+E^{*}\left(\frac{v_{i, j+3 / 2}^{n}-2 v_{i, j+1 / 2}^{n}+v_{i, j-1 / 2}^{n}}{\Delta \phi^{2}}\right. \\
+\frac{h^{*}\left(v_{i+1, j+1 / 2}^{n}-v_{i-1, j+1 / 2}^{n}\right)}{2 \Delta \theta}-h_{2}^{*} v_{i, j+1 / 2}^{n}=0, \\
\left.+\frac{v_{i+1, j+1 / 2}^{n}-2 v_{i, j+1 / 2}^{n}+v_{i-1, j+1 / 2}^{n}}{\Delta \theta^{2}}\right) \\
+\frac{v_{1}^{*}\left(u_{i+1 / 2, j+1}^{n}-u_{i+1 / 2, j-1}^{n}\right)}{2 \Delta \phi}+H^{*} \tau_{\theta}-\Phi v_{i, j+1 / 2}^{n}, \\
\frac{\left.h_{i+1, j}^{n}-h_{i-1, j}^{n}\right)}{2 \Delta \theta}+\frac{u_{1}^{*}\left(h_{i, j+1}^{n}-h_{i, j-1}^{n}\right)}{2 \Delta \phi}
\end{gathered}
$$

in which $u^{*}=u_{i+1 / 2, j}^{n} / \cos \theta_{i+1 / 2}, v^{*}=v_{i, j+1 / 2}^{n}, f^{*}=u_{i+1 / 2, j}^{n}$ $\tan \theta_{i+1 / 2}+(1 / \varepsilon) \sin \theta_{i}, F^{*}=F / \cos \theta_{i}, E^{*}=E / \varepsilon, E_{1}^{*}=$ $2 E \sin \theta_{i} / \varepsilon \cos ^{2} \theta_{i}, h^{*}=h_{i, j}^{n}, H^{*}=\alpha / \varepsilon h^{*}, \Phi=(\mu / \varepsilon)-$ $\left(E / \varepsilon \cos ^{2} \theta_{i+1 / 2}\right), h_{1}^{*}=h^{*} / \cos \theta_{i}$, and $h_{2}^{*}=h^{*} \tan \theta_{i}$.

3.2. The Leap-Frog Scheme. The Leap-frog differences are used for time derivatives and centered differences for space derivatives; the diffusion terms are lagged by one time step following the previous studies $[33,35]$; we obtain

$$
\begin{gathered}
\frac{u_{i+1 / 2, j}^{n+1}-u_{i+1 / 2, j}^{n-1}}{2 \Delta t}+\frac{u^{*}\left(u_{i+1 / 2, j+1}^{n}-u_{i+1 / 2, j-1}^{n}\right)}{2 \Delta \phi} \\
+\frac{v^{*}\left(u_{i+3 / 2, j}^{n}-u_{i-1 / 2, j}^{n}\right)}{2 \Delta \theta}-f^{*} v_{i, j+1 / 2}^{n} \\
=-\frac{F^{*}\left(h_{i, j+1}^{n}-h_{i, j-1}^{n}\right)}{2 \Delta \phi}
\end{gathered}
$$

$$
\begin{aligned}
& +E^{*}\left(\frac{u_{i+1 / 2, j+1}^{n-1}-2 u_{i+1 / 2, j}^{n-1}+u_{i+1 / 2, j-1}^{n-1}}{\Delta \phi^{2}}\right. \\
& \left.+\frac{u_{i+3 / 2, j}^{n-1}-2 u_{i+1 / 2, j}^{n-1}+u_{i-1 / 2, j}^{n-1}}{\Delta \theta^{2}}\right) \\
& -\frac{E_{1}^{*}\left(v_{i, j+3 / 2}^{n}-v_{i, j-1 / 2}^{n}\right)}{2 \Delta \phi}+H^{*} \tau_{\phi}-\Phi u_{i+1 / 2, j}^{n}, \\
& \frac{v_{i, j+1 / 2}^{n+1}-v_{i, j+1 / 2}^{n-1}}{2 \Delta t}+\frac{u^{*}\left(v_{i, j+3 / 2}^{n}-v_{i, j-1 / 2}^{n}\right)}{2 \Delta \phi} \\
& +\frac{v^{*}\left(v_{i+1, j+1 / 2}^{n}-v_{i-1, j+1 / 2}^{n}\right)}{2 \Delta \theta}+f^{*} u_{i+1 / 2, j}^{n} \\
& =-\frac{F\left(h_{i+1, j}^{n}-h_{i-1, j}^{n}\right)}{2 \Delta \theta} \\
& +E^{*}\left(\frac{v_{i, j+3 / 2}^{n-1}-2 v_{i, j+1 / 2}^{n-1}+v_{i, j-1 / 2}^{n-1}}{\Delta \phi^{2}}\right. \\
& \left.+\frac{v_{i+1, j+1 / 2}^{n-1}-2 v_{i, j+1 / 2}^{n-1}+v_{i-1, j+1 / 2}^{n-1}}{\Delta \theta^{2}}\right) \\
& +\frac{E_{1}^{*}\left(u_{i+1 / 2, j+1}^{n}-u_{i+1 / 2, j-1}^{n}\right)}{2 \Delta \phi}+H^{*} \tau_{\theta}-\Phi v_{i, j+1 / 2}^{n}, \\
& \frac{h_{i, j}^{n+1}-h_{i, j}^{n-1}}{2 \Delta t}+\frac{u^{*}\left(h_{i, j+1}^{n}-h_{i, j-1}^{n}\right)}{2 \Delta \phi} \\
& +\frac{v^{*}\left(h_{i+1, j}^{n}-h_{i-1, j}^{n}\right)}{2 \Delta \theta}+\frac{h_{1}^{*}\left(u_{i+1 / 2, j+1}^{n}-u_{i+1 / 2, j-1}^{n}\right)}{2 \Delta \phi} \\
& +\frac{h^{*}\left(v_{i+1, j+1 / 2}^{n}-v_{i-1, j+1 / 2}^{n}\right)}{2 \Delta \theta}-h_{2}^{*} v_{i, j+1 / 2}^{n}=0 .
\end{aligned}
$$

The Leap-frog method allows the direct calculation of $u_{i+1 / 2, j}^{n+1}, v_{i, j+1 / 2}^{n+1}, h_{i, j}^{n+1}$ from the known values at time levels $n$ and $n-1$, which are the explicit difference equations.

\section{Stability Analysis}

In this section we present the stability conditions of the finite difference numerical models by using the discrete Fourier analysis method and the Gerschgorin circle theorem. The specific analysis of procedure is given in the following parts.

First of all, we set $\mathbf{X}_{i, j}^{n}=\left(u_{i+1 / 2, j}^{n}, v_{i, j+1 / 2}^{n}, h_{i, j}^{n}\right)^{T}$, and after some rearrangement, (5) can be written as

$$
\begin{aligned}
\mathbf{X}_{i, j}^{n+1}= & A_{1} \mathbf{X}_{i+1, j}^{n}+C_{1} \mathbf{X}_{i-1, j}^{n}+B_{1} \mathbf{X}_{i, j}^{n} \\
& +D_{1} \mathbf{X}_{i, j+1}^{n}+E_{1} \mathbf{X}_{i, j-1}^{n}+\mathbf{e}_{n},
\end{aligned}
$$

in which $\mathbf{e}_{n}=\left(\Delta t H^{*} \tau_{\phi}, \Delta t H^{*} \tau_{\theta}, 0\right)^{T}$, 


$$
\begin{aligned}
& A_{1}=\left(\begin{array}{ccc}
-\frac{\Delta t v^{*}}{2 \Delta \theta}+\frac{\Delta t E^{*}}{\Delta \theta^{2}} & 0 & 0 \\
0 & -\frac{\Delta t v^{*}}{2 \Delta \theta}+\frac{\Delta t E^{*}}{\Delta \theta^{2}} & -\frac{\Delta t F}{2 \Delta \theta} \\
0 & -\frac{\Delta t h^{*}}{2 \Delta \theta} & -\frac{\Delta t v^{*}}{2 \Delta \theta}
\end{array}\right) \\
& C_{1}=\left(\begin{array}{ccc}
\frac{\Delta t v^{*}}{2 \Delta \theta}+\frac{\Delta t E^{*}}{\Delta \theta^{2}} & 0 & 0 \\
0 & \frac{\Delta t v^{*}}{2 \Delta \theta}+\frac{\Delta t E^{*}}{\Delta \theta^{2}} & \frac{\Delta t F}{2 \Delta \theta} \\
0 & \frac{\Delta t h^{*}}{2 \Delta \theta} & \frac{\Delta t v^{*}}{2 \Delta \theta}
\end{array}\right) \\
& B_{1}=\left(\begin{array}{ccc}
1-\Delta t \Phi-2 \Delta t E^{*}\left(\frac{1}{\Delta \phi^{2}}+\frac{1}{\Delta \theta^{2}}\right) & \Delta t f^{*} & 0 \\
-\Delta t f^{*} & 1-\Delta t \Phi-2 \Delta t E^{*}\left(\frac{1}{\Delta \phi^{2}}+\frac{1}{\Delta \theta^{2}}\right) & 0 \\
0 & \Delta t h_{2}^{*} & 1
\end{array}\right) \text {, } \\
& D_{1}=\left(\begin{array}{ccc}
-\frac{\Delta t u^{*}}{2 \Delta \phi}+\frac{\Delta t E^{*}}{\Delta \phi^{2}} & -\frac{\Delta t E_{1}^{*}}{2 \Delta \phi} & -\frac{\Delta t F^{*}}{2 \Delta \phi} \\
\frac{\Delta t E_{1}^{*}}{2 \Delta \phi} & -\frac{\Delta t u^{*}}{2 \Delta \phi}+\frac{\Delta t E^{*}}{\Delta \phi^{2}} & 0 \\
-\frac{\Delta t h_{1}^{*}}{2 \Delta \phi} & 0 & -\frac{\Delta t u^{*}}{2 \Delta \phi}
\end{array}\right) \\
& E_{1}=\left(\begin{array}{ccc}
\frac{\Delta t u^{*}}{2 \Delta \phi}+\frac{\Delta t E^{*}}{\Delta \phi^{2}} & \frac{\Delta t E_{1}^{*}}{2 \Delta \phi} & \frac{\Delta t F^{*}}{2 \Delta \phi} \\
-\frac{\Delta t E_{1}^{*}}{2 \Delta \phi} & \frac{\Delta t u^{*}}{2 \Delta \phi}+\frac{\Delta t E^{*}}{\Delta \phi^{2}} & 0 \\
\frac{\Delta t h_{1}^{*}}{2 \Delta \phi} & 0 & \frac{\Delta t u^{*}}{2 \Delta \phi}
\end{array}\right)
\end{aligned}
$$

In a similar fashion, (6) can be written as the following matrix equations:

$$
\begin{aligned}
\mathbf{X}_{i, j}^{n+1}= & A_{2} \mathbf{X}_{i-1, j}^{n}+B_{2} \mathbf{X}_{i+1, j}^{n}+C_{2} \mathbf{X}_{i, j-1}^{n} \\
& +D_{2} \mathbf{X}_{i, j+1}^{n}+E_{2} \mathbf{X}_{i, j}^{n}+F_{2} \mathbf{X}_{i+1, j}^{n-1}+H_{2} \mathbf{X}_{i-1, j}^{n-1} \\
& +M_{2} \mathbf{X}_{i, j+1}^{n-1}+N_{2} \mathbf{X}_{i, j-1}^{n-1}+P_{2} \mathbf{X}_{i, j}^{n-1}+\mathbf{e}_{n},
\end{aligned}
$$

where $\mathbf{e}_{n}=\left(2 \Delta t H^{*} \tau_{\phi}, 2 \Delta t H^{*} \tau_{\theta}, 0\right)^{T}$,

$$
A_{2}=-B_{2}=\left(\begin{array}{ccc}
\frac{\Delta t v^{*}}{\Delta \theta} & 0 & 0 \\
0 & \frac{\Delta t v^{*}}{\Delta \theta} & \frac{\Delta t F}{\Delta \theta} \\
0 & \frac{\Delta t h^{*}}{\Delta \theta} & \frac{\Delta t v^{*}}{\Delta \theta}
\end{array}\right),
$$

$$
C_{2}=-D_{2}=\left(\begin{array}{ccc}
\frac{\Delta t u^{*}}{\Delta \phi} & \frac{\Delta t E_{1}^{*}}{\Delta \phi} & \frac{\Delta t F^{*}}{\Delta \phi} \\
-\frac{\Delta t E_{1}^{*}}{\Delta \phi} & \frac{\Delta t u^{*}}{\Delta \phi} & 0 \\
\frac{\Delta t h_{1}^{*}}{\Delta \phi} & 0 & \frac{\Delta t u^{*}}{\Delta \phi}
\end{array}\right) \text {, }
$$

$$
E_{2}=\left(\begin{array}{ccc}
-2 \Delta t \Phi & 2 \Delta t f^{*} & 0 \\
-2 \Delta t f^{*} & -2 \Delta t \Phi & 0 \\
0 & 2 \Delta t h_{2}^{*} & 0
\end{array}\right) \text {, }
$$$$
F_{2}=H_{2}=\left(\begin{array}{ccc}
\frac{2 \Delta t E^{*}}{\Delta \theta^{2}} & 0 & 0 \\
0 & \frac{2 \Delta t E^{*}}{\Delta \theta^{2}} & 0 \\
0 & 0 & 0
\end{array}\right) \text {, }
$$ 


$$
\begin{gathered}
M_{2}=N_{2}=\left(\begin{array}{ccc}
\frac{2 \Delta t E^{*}}{\Delta \phi^{2}} & 0 & 0 \\
0 & \frac{2 \Delta t E^{*}}{\Delta \phi^{2}} & 0 \\
0 & 0 & 0
\end{array}\right), \\
=\left(\begin{array}{ccc}
1-4 \Delta t E^{*}\left(\frac{1}{\Delta \phi^{2}}+\frac{1}{\Delta \theta^{2}}\right) & 0 \\
0 & 1-4 \Delta t E^{*}\left(\frac{1}{\Delta \phi^{2}}+\frac{1}{\Delta \theta^{2}}\right) & 0 \\
0 & 0 & 1
\end{array}\right) .
\end{gathered}
$$

Definition 1. The two-dimensional discrete Fourier transform of $u \in \ell_{2}$ is the function $u^{\dagger} \in L_{2}([-\pi, \pi] \times[-\pi, \pi])$ defined by (see [39])

$$
u^{\dagger}(\xi, \beta)=\frac{1}{2 \pi} \sum_{m, k=-\infty}^{\infty} \exp (-i(m \xi+k \beta)) u_{m, k}
$$

for $\xi, \beta \in[-\pi, \pi]$.

We begin by taking the discrete Fourier transform of both sides of (7), and we can obtain

$$
\begin{aligned}
& \mathbf{X}^{\dagger n+1}(\xi, \beta)=\frac{1}{2 \pi} \sum_{m, k=-\infty}^{\infty} \exp (-i(m \xi+k \beta)) \mathbf{X}_{m, k}^{n+1} \\
& =\frac{1}{2 \pi} \sum_{m, k=-\infty}^{\infty} \exp (-i(m \xi+k \beta)) \\
& \times\left(A_{1} \mathbf{X}_{m+1, k}^{n}+C_{1} \mathbf{X}_{m-1, k}^{n}+B_{1} \mathbf{X}_{m, k}^{n}\right. \\
& \left.+D_{1} \mathbf{X}_{m, k+1}^{n}+E_{1} \mathbf{X}_{m, k-1}^{n}+\mathbf{e}_{n}\right) \\
& =\frac{A_{1}}{2 \pi} \sum_{m, k=-\infty}^{\infty} \exp (-i(m \xi+k \beta)) \mathbf{X}_{m+1, k}^{n} \\
& +\frac{C_{1}}{2 \pi} \sum_{m, k=-\infty}^{\infty} \exp (-i(m \xi+k \beta)) \mathbf{X}_{m-1, k}^{n} \\
& +\frac{B_{1}}{2 \pi} \sum_{m, k=-\infty}^{\infty} \exp (-i(m \xi+k \beta)) \mathbf{X}_{m, k}^{n} \\
& +\frac{D_{1}}{2 \pi} \sum_{m, k=-\infty}^{\infty} \exp (-i(m \xi+k \beta)) \mathbf{X}_{m, k+1}^{n} \\
& +\frac{E_{1}}{2 \pi} \sum_{m, k=-\infty}^{\infty} \exp (-i(m \xi+k \beta)) \mathbf{X}_{m, k-1}^{n} \\
& +\frac{1}{2 \pi} \sum_{m, k=-\infty}^{\infty} \exp (-i(m \xi+k \beta)) \mathbf{e}_{n}
\end{aligned}
$$

By making the change of variables $l=m \pm 1$ we get

$$
\begin{aligned}
& \frac{1}{2 \pi} \sum_{m, k=-\infty}^{\infty} \exp (-i(m \xi+k \beta)) \mathbf{X}_{m \pm 1, k}^{n} \\
& \quad=\frac{1}{2 \pi} \sum_{l, k=-\infty}^{\infty} \exp [-i((l \mp 1) \xi+k \beta)] \mathbf{X}_{l, k}^{n} \\
& \quad=\exp ( \pm i \xi) \frac{1}{2 \pi} \sum_{l, k=-\infty}^{\infty} \exp (-i(l \xi+k \beta)) \mathbf{X}_{l, k}^{n} \\
& \quad=\exp ( \pm i \xi) \mathbf{X}^{\dagger n}(\xi, \beta) .
\end{aligned}
$$

Similarly, we have

$$
\frac{1}{2 \pi} \sum_{m, k=-\infty}^{\infty} \exp (-i(m \xi+k \beta)) \mathbf{X}_{m, k \pm 1}^{n}=\exp ( \pm i \beta) \mathbf{X}^{\dagger n}(\xi, \beta)
$$

Thus using the expressions (13)-(14) in (12) leads to

$$
\mathbf{X}^{\dagger n+1}(\xi, \beta)=G_{1}(\xi, \beta) \mathbf{X}^{\dagger n}(\xi, \beta)+\mathbf{e}_{n}^{\prime},
$$

where $\mathbf{e}_{n}^{\prime}=(1 / 2 \pi) \sum_{m, k=-\infty}^{\infty} \exp (-i(m \xi+k \beta)) \mathbf{e}_{n}$, and the growth matrix

$$
\begin{aligned}
G_{1}(\xi, \beta) & \\
= & A_{1} \exp (i \xi)+C_{1} \exp (-i \xi)+B_{1} \\
& +D_{1} \exp (i \beta)+E_{1} \exp (-i \beta) \\
= & \left(\begin{array}{lll}
a_{11} & a_{12} & a_{13} \\
a_{21} & a_{22} & a_{23} \\
a_{31} & a_{32} & a_{33}
\end{array}\right),
\end{aligned}
$$

in which

$$
\begin{gathered}
a_{11}=a_{22}=1-\Delta t \Phi-2 \Delta t E^{*}\left(\frac{1}{\Delta \phi^{2}}+\frac{1}{\Delta \theta^{2}}\right) \\
+2 \Delta t E^{*}\left(\frac{\cos \beta}{\Delta \phi^{2}}+\frac{\cos \xi}{\Delta \theta^{2}}\right)-i \Delta t\left(\frac{u^{*} \sin \beta}{\Delta \phi}+\frac{v^{*} \sin \xi}{\Delta \theta}\right), \\
a_{12}=\Delta t f^{*}-\frac{i \Delta t E_{1}^{*}}{\Delta \phi} \sin \beta, \quad a_{13}=\frac{-i \Delta t F^{*}}{\Delta \phi} \sin \beta, \\
a_{21}=-\Delta t f^{*}+\frac{i \Delta t E_{1}^{*}}{\Delta \phi} \sin \beta, \quad a_{23}=\frac{-i \Delta t F}{\Delta \theta} \sin \xi, \\
a_{31}=-\frac{i \Delta t h_{1}^{*}}{\Delta \phi} \sin \beta, \quad a_{32}=\Delta t h_{2}^{*}-\frac{i \Delta t h^{*}}{\Delta \theta} \sin \xi, \\
a_{33}=1-i \Delta t\left(\frac{u^{*}}{\Delta \phi} \sin \beta+\frac{v^{*}}{\Delta \theta} \sin \xi\right) .
\end{gathered}
$$

Remark 2. The discrete Fourier transform is used to deal with the vector $\mathbf{X}_{i, j}^{n}=\left(u_{i+1 / 2, j}^{n}, v_{i, j+1 / 2}^{n}, h_{i, j}^{n}\right)^{T}$. Therefore, the individual variables $u^{*}, v^{*}$, and $h^{*}$ in the coefficient matrix of 
expression (7) can be treated as constants. Consequently, the coefficient matrixes can be extracted from the Fourier transform in the expression (12). These are similar to the frozen coefficients approach for discussing the stability of numerical solution of variable coefficient partial differential equation (see [40-42]).

Definition 3. The three-dimensional discrete Fourier transform of $u \in \ell_{2}$ is the function $u^{\dagger} \in L_{2}([-\pi, \pi] \times[-\pi, \pi] \times$ $[-\pi, \pi])$ defined by

$$
u^{\dagger}(\xi, \beta, \varphi)=\frac{1}{2 \pi} \sum_{m, k, n=-\infty}^{\infty} \exp (-i(m \xi+k \beta+n \varphi)) u_{m, k}^{n}
$$

for $\xi, \beta, \varphi \in[-\pi, \pi]$.

Taking the discrete Fourier transform (18) to the both sides of (9), similar to the derivation of (15), we have

$$
\mathbf{X}^{\dagger n+1}(\xi, \beta, \varphi)=G_{2}(\xi, \beta, \varphi) \mathbf{X}^{\dagger n}(\xi, \beta, \varphi)+\mathbf{e}_{n}^{\prime}
$$

where the growth matrix

$$
\begin{aligned}
G_{2}(\xi, \theta, \beta)= & A_{2} \exp (-i \xi)+B_{2} \exp (i \xi)+C_{2} \exp (-i \beta) \\
& +D_{2} \exp (i \beta)+E_{2}+F_{2} \exp [i(\xi-\varphi)] \\
& +H_{2} \exp [-i(\xi+\varphi)]+M_{2} \exp [i(\beta-\varphi)] \\
& +N_{2} \exp [-i(\beta+\varphi)]+P_{2} \exp (-i \varphi) \\
= & \left(\begin{array}{lll}
b_{11} & b_{12} & b_{13} \\
b_{21} & b_{22} & b_{23} \\
b_{31} & b_{32} & b_{33}
\end{array}\right),
\end{aligned}
$$

in which

$$
\begin{aligned}
b_{11}= & b_{22}=-2 \Delta t \Phi-2 i \Delta t\left(\frac{u^{*}}{\Delta \phi} \sin \beta+\frac{v^{*}}{\Delta \theta} \sin \xi\right) \\
& +\left[1-4 \Delta t E^{*}\left(\frac{1}{\Delta \phi^{2}}+\frac{1}{\Delta \theta^{2}}\right)\right] \exp (-i \varphi) \\
& +\frac{2 \Delta t E^{*}}{\Delta \phi^{2}}[\exp (i(\beta-\varphi))+\exp (-i(\beta+\varphi))] \\
& +\frac{2 \Delta t E^{*}}{\Delta \theta^{2}}[\exp (i(\xi-\varphi))+\exp (-i(\xi+\varphi))] \\
b_{13}=- & \frac{2 i \Delta t F^{*}}{\Delta \phi} \sin \beta, \quad b_{21}=-2 \Delta t f^{*}+\frac{2 i \Delta t E_{1}^{*}}{\Delta \phi} \sin \beta, \\
b_{23} & =-\frac{2 i \Delta t F}{\Delta \theta} \sin \xi, \quad b_{31}=-\frac{2 i \Delta t h_{1}^{*}}{\Delta \phi} \sin \beta,
\end{aligned}
$$

$$
\begin{gathered}
b_{32}=2 \Delta t h_{2}^{*}-\frac{2 i \Delta t h^{*}}{\Delta \theta} \sin \xi \\
b_{33}=\exp (-i \varphi)-2 i \Delta t\left(\frac{u^{*}}{\Delta \phi} \sin \beta+\frac{v^{*}}{\Delta \theta} \sin \xi\right) .
\end{gathered}
$$

Theorem 4. The difference schemes (7) and (9) are stable in the $\ell_{2}$ norm; then there exist constants $\Delta x_{0}, \Delta y_{0}, \Delta t_{0}$, and $C$, independent of $\Delta x, \Delta y$, and $\Delta t$, so that

$$
|\rho(G)| \leq 1+C \Delta t
$$

for $0<\Delta t \leq \Delta t_{0}$, and $0<\Delta x \leq \Delta x_{0}, 0<\Delta y \leq \Delta y_{0}$, and for all $\xi, \theta, \varphi \in[-\pi, \pi] . \rho(G)$ is the spectral radius of the matrix $G$ $\left(G_{1}\right.$ and $\left.G_{2}\right)$ [39].

Theorem 5 (Gerschgorin circle theorem, see [39]). Suppose $A=\left(a_{i j}\right)$ is a general $n \times n$ matrix, and $\rho_{s}=\sum_{j=1, j \neq s}^{n}\left|a_{s j}\right|$ is the sum of the absolute values of the elements in the sth row except for the diagonal element. For each eigenvalue $\lambda$ of $A$, there exists an such that

$$
\left|\lambda-a_{s s}\right| \leq \rho_{s}, \quad s=1,2, \ldots n .
$$

If $\lambda$ is an eigenvalue of $G_{1}$, by using Gerschgorin circle theorem and the triangular inequality, one has

$$
\begin{aligned}
& |\lambda|-\left|a_{11}\right| \leq\left|a_{12}\right|+\left|a_{13}\right|, \\
& |\lambda|-\left|a_{22}\right| \leq\left|a_{21}\right|+\left|a_{23}\right|, \\
& |\lambda|-\left|a_{33}\right| \leq\left|a_{31}\right|+\left|a_{32}\right| .
\end{aligned}
$$

Then the time-space steps size and model parameters yield the following conditions (one takes $C \leq \epsilon / \Delta t$, where $0<\epsilon \leq 1$ ):

$$
\begin{gathered}
\Delta t \leq \frac{\epsilon}{\left|f^{*}\right|+|\Phi|}, \quad \Delta t \leq \frac{\epsilon}{\left|h_{2}^{*}\right|} \quad(\xi=\beta=0), \\
\Delta t\left(\left|f^{*}\right|+|\Phi|+\frac{4\left|E^{*}\right|}{\Delta \phi^{2}}+\frac{4\left|E^{*}\right|}{\Delta \theta^{2}}\right) \leq \epsilon \quad(\xi=\beta=\pi),
\end{gathered}
$$

$$
\begin{gathered}
\frac{\Delta t}{\Delta \phi}\left(\left|u^{*}\right|+\left|E_{1}^{*}\right|+\left|F^{*}\right|\right)+\frac{\Delta t\left|v^{*}\right|}{\Delta \theta}+\Delta t\left(|\Phi|+\left|f^{*}\right|\right) \\
+\frac{2 \Delta t\left|E^{*}\right|}{\Delta \phi^{2}}+\frac{2 \Delta t\left|E^{*}\right|}{\Delta \theta^{2}} \leq \epsilon,
\end{gathered}
$$

$$
\begin{aligned}
& \frac{\Delta t}{\Delta \phi}\left(\left|u^{*}\right|+\left|E_{1}^{*}\right|\right)+\frac{\Delta t}{\Delta \theta}\left(\left|v^{*}\right|+|F|\right)+\Delta t\left(|\Phi|+\left|f^{*}\right|\right) \\
& +\frac{2 \Delta t\left|E^{*}\right|}{\Delta \phi^{2}}+\frac{2 \Delta t\left|E^{*}\right|}{\Delta \theta^{2}} \leq \epsilon, \\
& \frac{\Delta t}{\Delta \phi}\left(\left|u^{*}\right|+\left|h_{1}^{*}\right|\right)+\frac{\Delta t}{\Delta \theta}\left(\left|v^{*}\right|+\left|h^{*}\right|\right)+\Delta t\left|h_{2}^{*}\right| \leq \epsilon \\
& \quad\left(\xi=\beta=\frac{\pi}{2}\right) .
\end{aligned}
$$




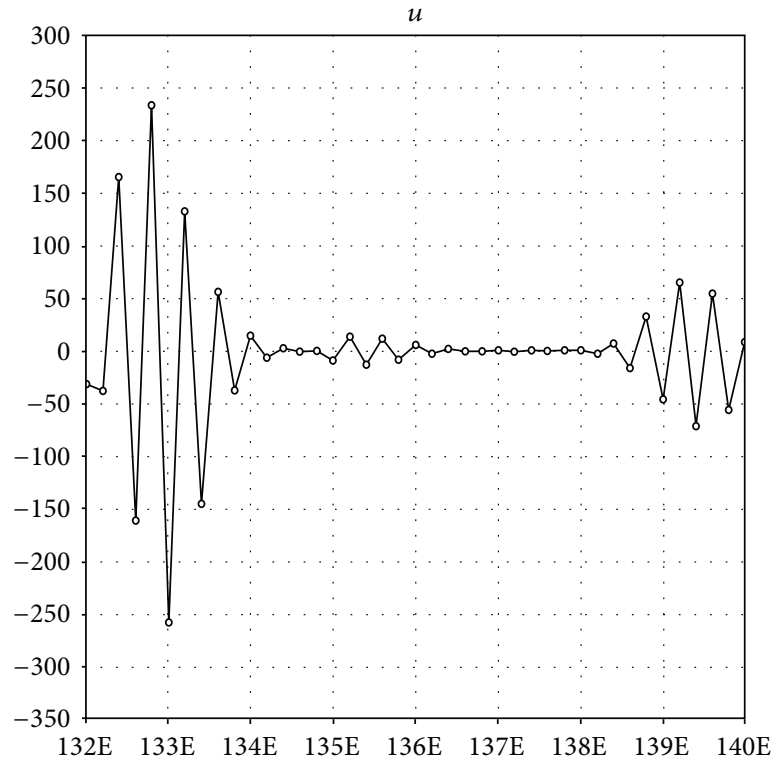

(a)

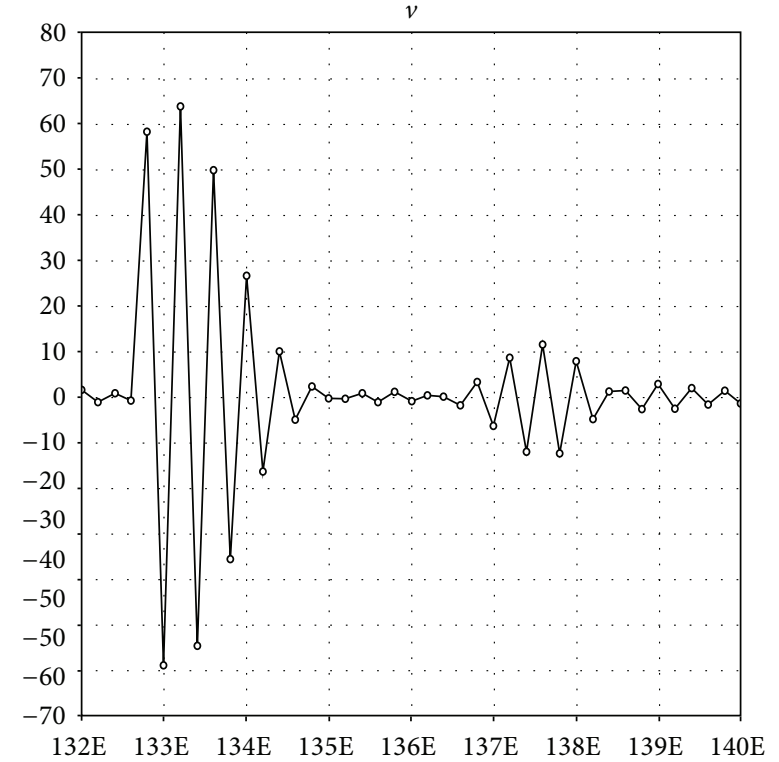

(b)

FIgURE 2: The figures show the values of the zonal velocity $u$ and the meridional velocity $v$ at the latitude $30^{\circ} \mathrm{N}$ after running the model with 30 steps.

Therefore, one has $|\lambda| \leq 1+C \Delta t$, and the FTCS scheme is conditionally stable. The condition is as follows:

$$
\Delta t \leq \min \left\{\frac{\epsilon}{\left|f^{*}\right|+|\Phi|}, \frac{\epsilon}{\left|h_{2}^{*}\right|}, \frac{\epsilon}{\delta_{1}}, \frac{\epsilon}{\delta_{2}}, \frac{\varepsilon}{\delta_{3}}, \frac{\epsilon}{\delta_{4}}\right\},
$$

in which $\delta_{1}=\left|f^{*}\right|+|\Phi|+\left(4\left|E^{*}\right| / \Delta \phi^{2}\right)+\left(4\left|E^{*}\right| / \Delta \theta^{2}\right), \delta_{2}=$ $(1 / \Delta \phi)\left(\left|u^{*}\right|+\left|E_{1}^{*}\right|+\left|F^{*}\right|\right)+\left(\left|v^{*}\right| / \Delta \theta\right)+|\Phi|+\left|f^{*}\right|+\left(2\left|E^{*}\right| / \Delta \phi^{2}\right)+$ $\left(2\left|E^{*}\right| / \Delta \theta^{2}\right), \delta_{3}=(1 / \Delta \phi)\left(\left|u^{*}\right|+\left|E_{1}^{*}\right|\right)+(1 / \Delta \theta)\left(\left|v^{*}\right|+|F|\right)+$ $|\Phi|+\left|f^{*}\right|+\left(2\left|E^{*}\right| / \Delta \phi^{2}\right)+\left(2\left|E^{*}\right| / \Delta \theta^{2}\right)$, and $\delta_{4}=(1 / \Delta \phi)\left(\left|u^{*}\right|+\right.$ $\left.\left|h_{1}^{*}\right|\right)+(1 / \Delta \theta)\left(\left|v^{*}\right|+\left|h^{*}\right|\right)+\left|h_{2}^{*}\right|$.

Similarly, one obtains the stable condition of the explicit Leap-frog finite-difference scheme (9):

$$
\Delta t \leq \min \left\{\frac{\epsilon}{2\left(\left|f^{*}\right|+|\Phi|\right)}, \frac{\epsilon}{2\left|h_{2}^{*}\right|}, \frac{\epsilon}{2 \delta_{1}}, \frac{\epsilon}{2 \delta_{2}}, \frac{\epsilon}{2 \delta_{3}}, \frac{\epsilon}{2 \delta_{4}}\right\},
$$

where $\delta_{1}, \delta_{2}, \delta_{3}$, and $\delta_{4}$ are the same as in expression (28).

Remark 6 . The derivations of stability conclusions in this study are still valid for both A-grid and B-grid; the results depend mainly on the choice of vector $\mathbf{X}_{i, j}^{n}$; for example, in the A-grid, we take $\mathbf{X}_{i, j}^{n}=\left(u_{i, j}^{n}, v_{i, j}^{n}, h_{i, j}^{n}\right)^{T}$. In addition, it is easy to prove that the stability conditions derived from $\mathrm{C}$-grid are the same for both A-grid and B-grid.

As a matter of fact, when the rotation, eddy viscosity, wind stress, and interfacial friction are neglected, the second expression in (27) can be written as $(\epsilon=1)$

$$
\frac{\Delta t\left|u^{*}\right|}{\Delta \phi}+\frac{\Delta t\left|v^{*}\right|}{\Delta \theta}+\frac{\Delta t|F|}{\Delta \theta} \leq 1
$$

TABLE 1: The standard values of parameters in the model.

\begin{tabular}{lc}
\hline Parameter & Value \\
\hline$r_{0}$ & $6.37 \times 10^{6} \mathrm{~m}$ \\
$H$ & $500 \mathrm{~m}$ \\
$\tau_{0}$ & $0.1 \mathrm{~Pa}$ \\
$U$ & $0.1 \mathrm{~m} \mathrm{~s}^{-1}$ \\
$\gamma$ & $4.3752 \times 10^{-8} \mathrm{~s}^{-1}$ \\
$g$ & $9.8 \mathrm{~m} \mathrm{~s}^{-2}$ \\
$\rho$ & $1023.5 \mathrm{~kg} \mathrm{~m}^{-3}$ \\
$A_{H}$ & $450 \mathrm{~m}^{2} \mathrm{~s}^{-1}$ \\
$\omega$ & $7.292 \times 10^{-5} \mathrm{~s}^{-1}$ \\
$g^{\prime}$ & $0.044 \mathrm{~m} \mathrm{~s}^{-2}$ \\
\hline
\end{tabular}

This is the Courant-Friedrichs-Lewy condition (CFL condition; see $[13,34,42])$ in two-dimensional case. Assuming the terms $\left|f^{*}\right|=0,|\Phi|=0$ and $\epsilon=1$ in the expression (26), then we have

$$
\frac{4 \Delta t\left|E^{*}\right|}{\Delta \phi^{2}}+\frac{4 \Delta t\left|E^{*}\right|}{\Delta \theta^{2}} \leq 1
$$

In fact, this is the same as the conditions identified by Blumberg and Mellor in 1987 [43]. When the rotation, wind stress and interfacial friction terms are neglected and set $\epsilon=$ 1 , the first and second expressions in (27) are given as

$$
\frac{\Delta t\left|u^{*}\right|}{\Delta \phi}+\frac{\Delta t\left|v^{*}\right|}{\Delta \theta}+\frac{1}{2}\left(\frac{\Delta t\left|E^{*}\right|}{\Delta \phi^{2}}+\frac{\Delta t\left|E^{*}\right|}{\Delta \theta^{2}}\right) \leq 1 .
$$

This condition is the same as $[15,20]$ that given by Casulli and Cheng. The stability criteria (30)-(32) have been widely 


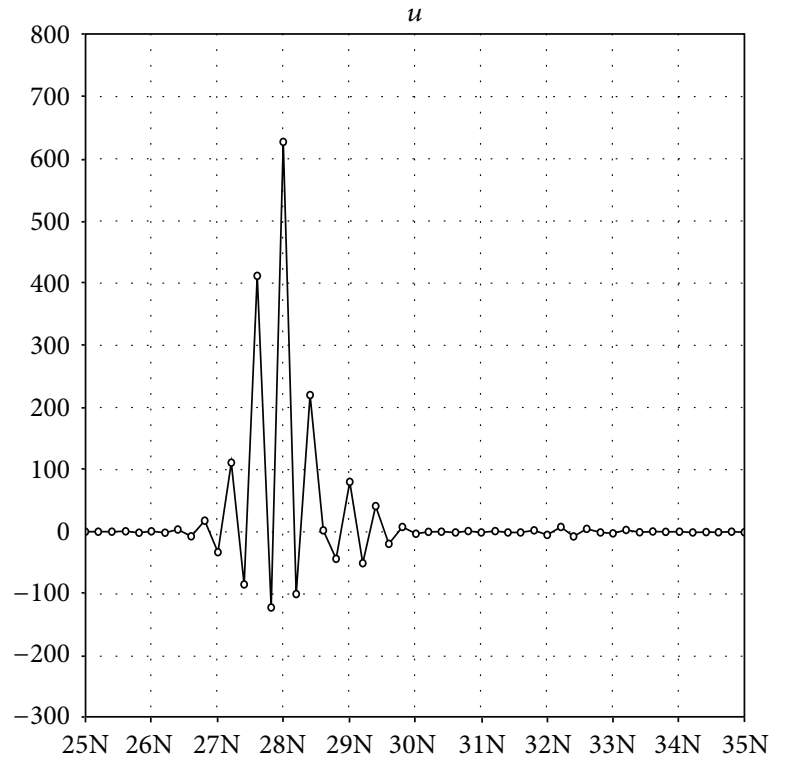

(a)

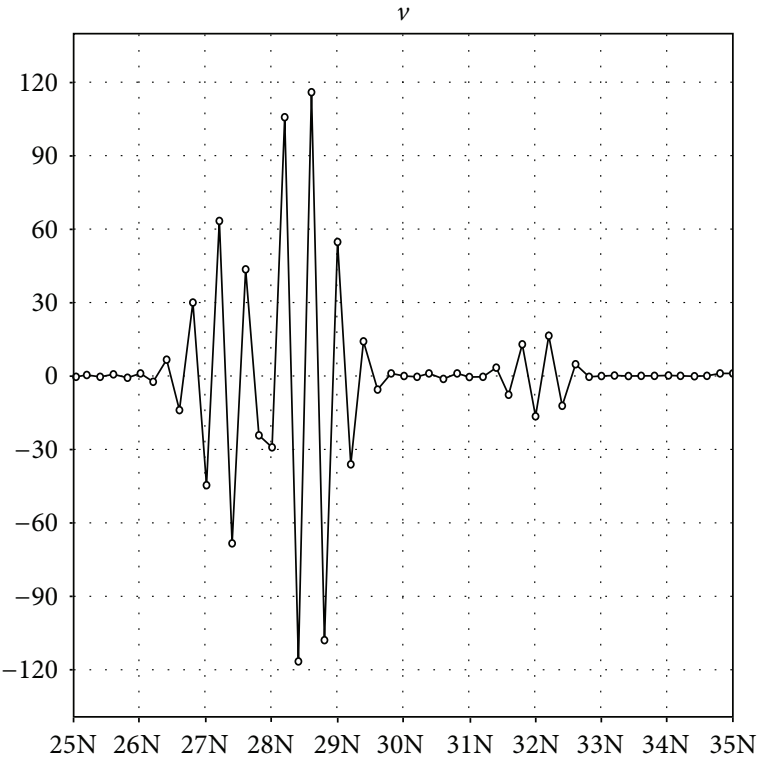

(b)

Figure 3: The figures show the values of the zonal velocity $u$ and the meridional velocity $v$ at the longitude $135^{\circ} \mathrm{E}$ after running the model with 30 steps.

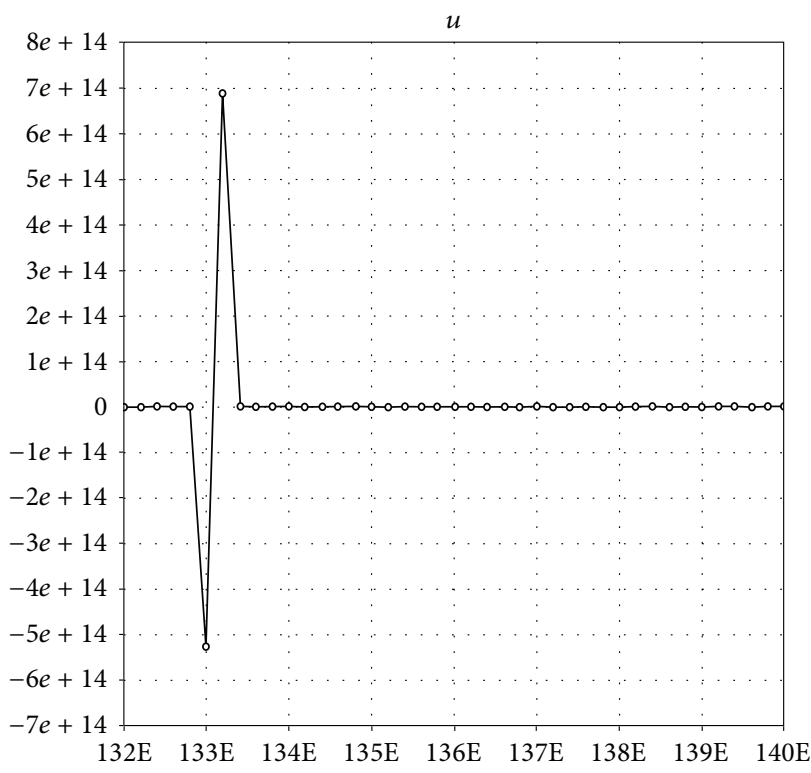

(a)

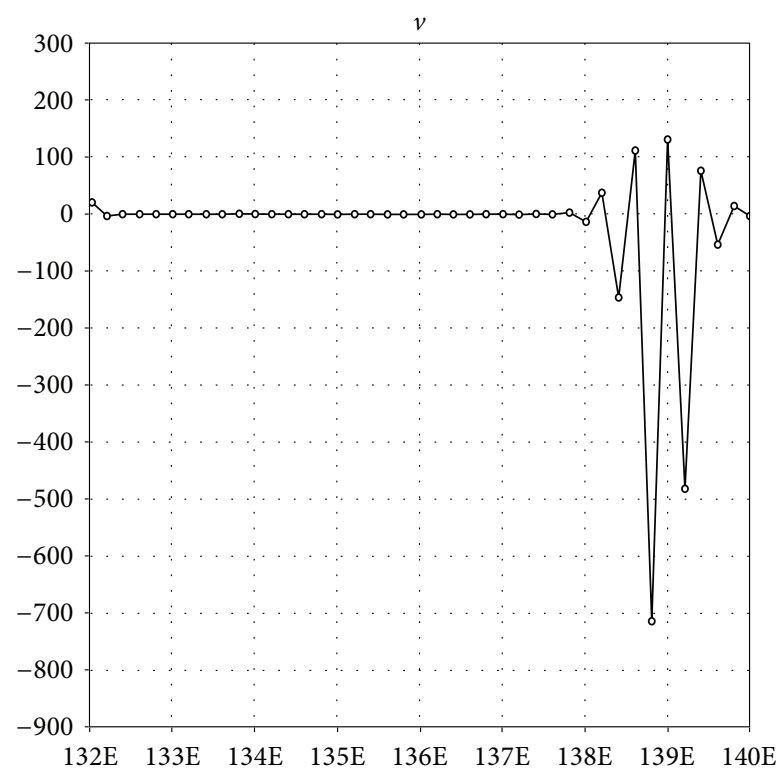

(b)

FIGURE 4: The figures show the values of the zonal velocity $u$ and the meridional velocity $v$ at the latitude $29^{\circ} \mathrm{N}$ after a time integration of 5 hours.

applied to the numerical model for the selection of the timestep size. However, these three conditions are only special cases in our results.

\section{Numerical Experiments}

In this section, numerical examples are given to test our results. In the present study, we take the FTCS scheme, for example (because the stability criterions of the Leap-frog finite-difference scheme are similar to the FTCS scheme). The domain of integration is set as a part of the North Pacific basin $\left(25^{\circ}-35^{\circ} \mathrm{N}, 132^{\circ}-140^{\circ} \mathrm{E}\right)$. We use a realistic coastline and the $200 \mathrm{~m}$ depth contour as the continental boundary [27]. The horizontal resolution is $0.2^{\circ} \times 0.2^{\circ}$; that is, the space-step size $\Delta \theta=\Delta \phi=0.2$. Standard parameter values in the shallowwater model are shown in Table 1. 


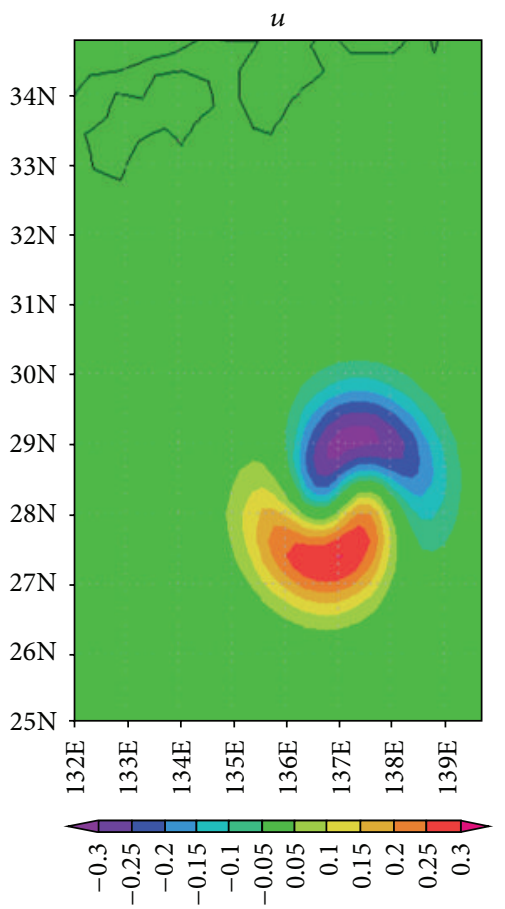

(a)

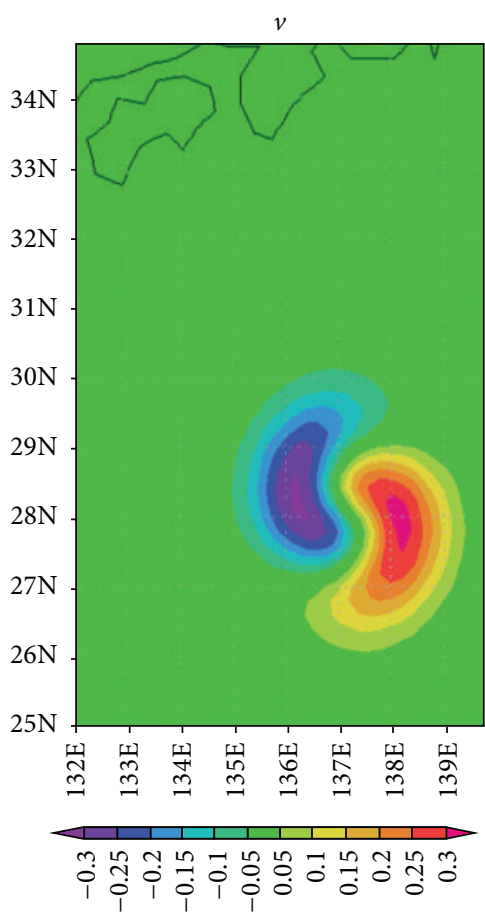

(b)

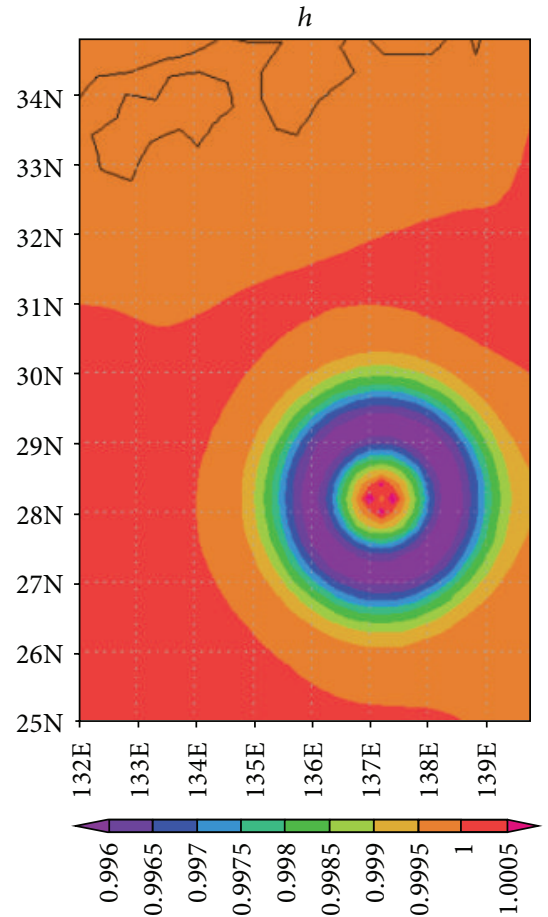

(c)

FIGURE 5: The figures show the values of the zonal velocity $u$, the meridional velocity $v$, and the layer thickness $h$ after a time integration of 8 hours.

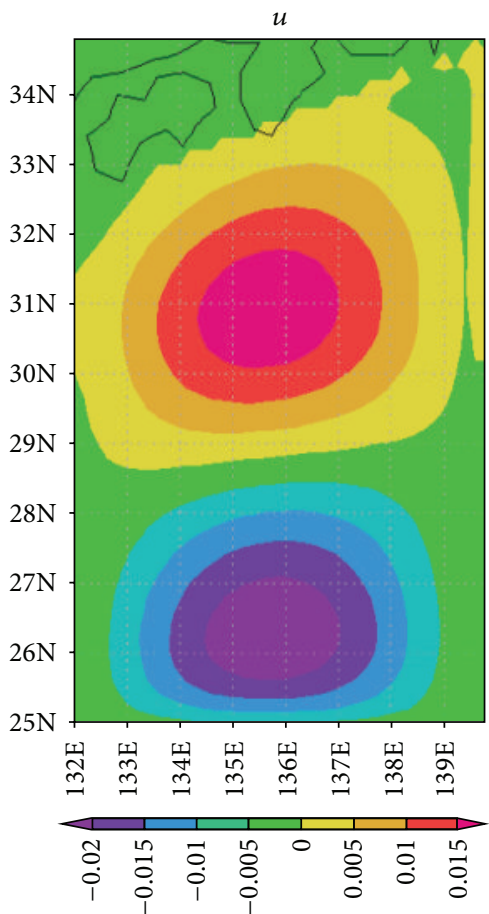

(a)

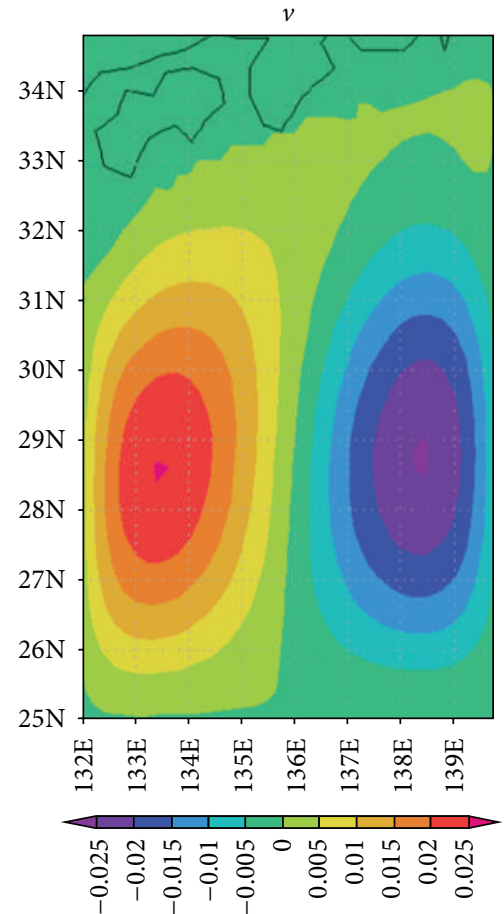

(b)

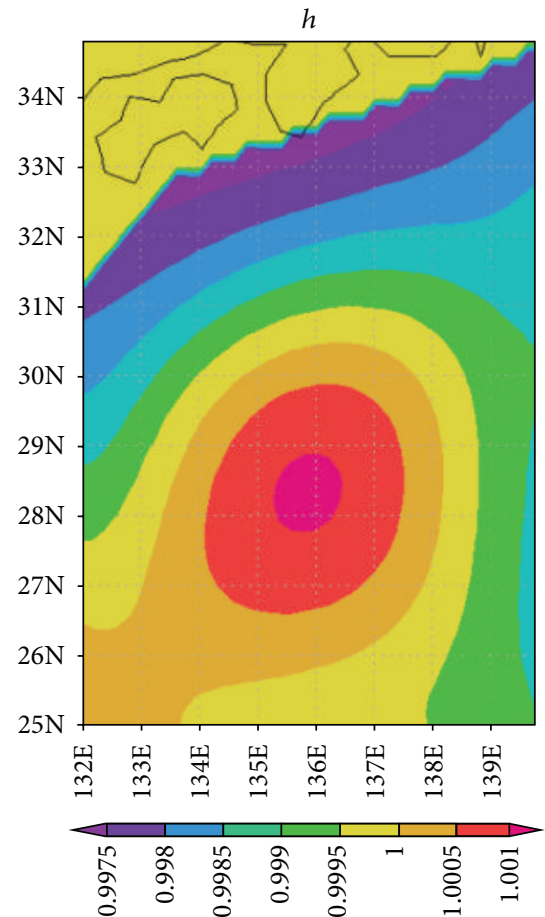

(c)

FIgURE 6: The figures show the values of the zonal velocity $u$, the meridional velocity $v$, and the layer thickness $h$ after a time integration of 8 years. 

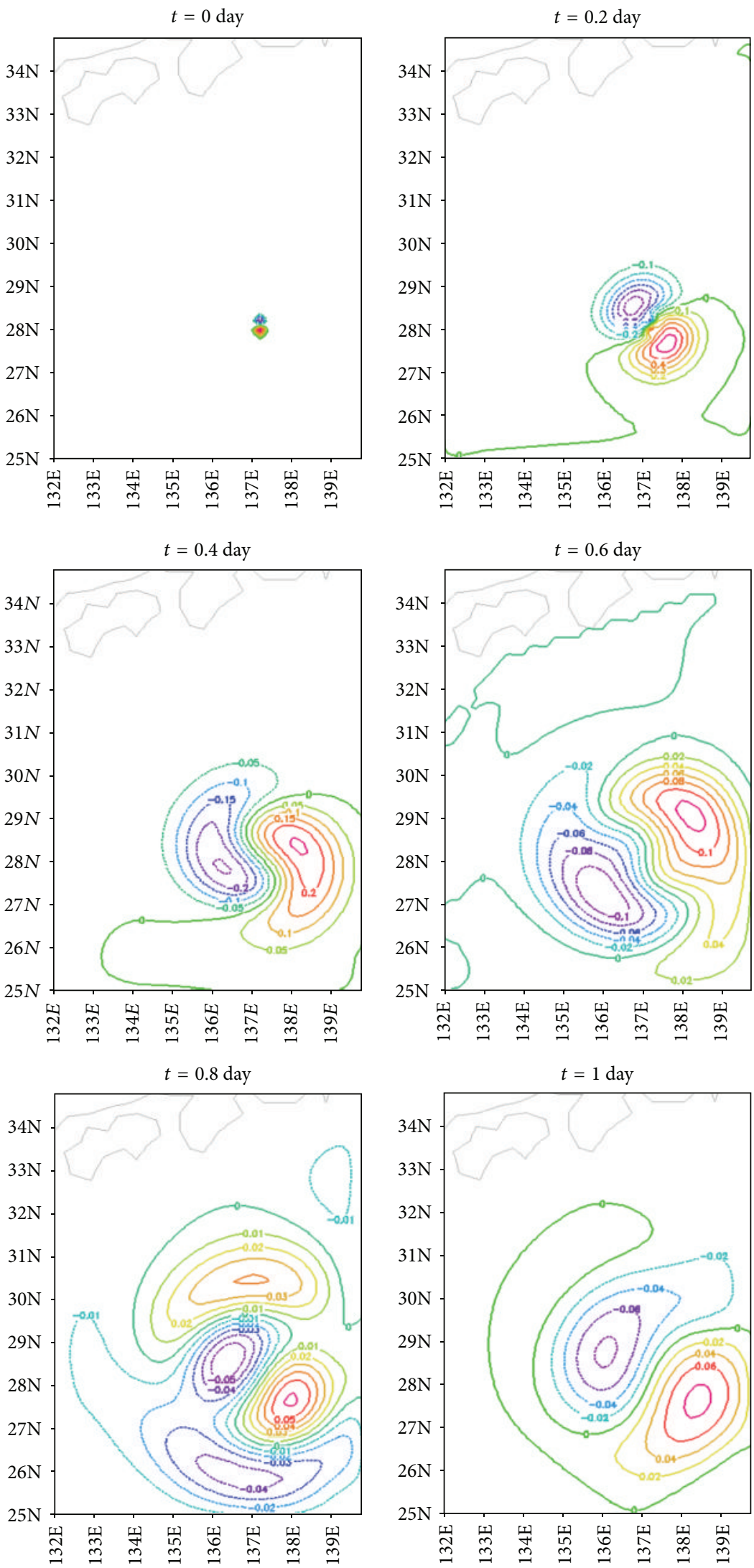

FIGURE 7: The figures show the meridional velocity of the ocean current throughout a period of the time-dependent solution that evolves in one day. 
5.1. Example 1. We take $\epsilon=0.3$, the zonal velocity $u=0.3$, and the meridional velocity $v=0.3$. According to the expression (28), it is easy to obtain $\Delta t<3 / 233020$; multiplying by the time scale, we have $\Delta t<821 \mathrm{~s}$.

In the light of the CFL condition (30), we have $\Delta t<$ 5790 s.

With the expression (31), we obtain $\Delta t<4.6 \times 10^{8} \mathrm{~s}$.

From the stability criterion (32), we get $\Delta t<2.2 \times$ $10^{7} \mathrm{~s}$.

Case 1. Setting a time-step size $\Delta t=825 \mathrm{~s}$, after running the model with 30 steps (55/8 hours), Figure 2 gives the values (the dimensionless quantity, as well as the following results) of the zonal velocity $u$ and the meridional velocity $v$ at the latitude $30^{\circ} \mathrm{N}$; Figure 3 gives the results at the longitude $135^{\circ} \mathrm{E}$. It is not difficult to find that the current velocities $u$ and $v$ are not in accord with the actual condition of ocean.

Case 2. When we choose the step size $\Delta t=1200 \mathrm{~s}$, the results in Figure 4 give the values of the zonal velocity $u$ and the meridional velocity $v$ at the latitude $29^{\circ} \mathrm{N}$ after a time integration of 5 hours. Obviously, the results are also unreasonable. Moreover, after continuing the calculation of model, we find that the results start to overflow after running the model with 17 steps (17/3 hours).

Case 3. The model is run with a time step size of $\Delta t=300 \mathrm{~s}$, Figures 5 and 6 show the values of the zonal velocity $u$, the meridional velocity $v$, and the layer thickness $h$ after a time integration of 8 hours and 8 years, respectively. These results illustrate that the model is integrated for long periods of time, and the results are still reasonable.

It is obvious that the stability condition (28) is reasonable, because the numerical model is unstable when we take the time step size $\Delta t>821 \mathrm{~s}$ (as shown in Cases 1 and 2). On the other hand, it is easy to see that our results are more strict and accurate than the CFL condition (30) and other two criterions (31) and (32).

5.2. Example 2. In this example, the 1.5-layer shallow-water numerical model that is designed by us is used to simulate the ocean current. Based on Example 1, the ocean basin is also adopted with the part of the North Pacific basin $\left(25^{\circ}-\right.$ $35^{\circ} \mathrm{N}, 132^{\circ}-140^{\circ} \mathrm{E}$ ). The time-space step sizes and the standard values of parameters in the model are the same as Case 3 in Example 1. Figure 7 gives the meridional velocity of the ocean current throughout a period of the time-dependent solution that evolves in one day. We will make an attempt to use this explicit shallow-water numerical model to simulate the Kuroshio current and its extension system in further studies.

\section{Conclusions}

The FTCS and the Leap-frog finite difference scheme for solving 1.5-layer shallow-water equations in spherical coordinates have been presented. The stability conditions of these two types of difference schemes are given, which include the CFL condition and other two criterions $[15,20,43]$. The numerical experiments are proposed for testing the stability of the FTCS scheme; the numerical results illustrate that our stability conditions are effective and reasonable. Moreover, the present stability criterion is shown to be more accurate than other criterions that this research mentioned. The theory of stability analysis in this paper can also be used to study the complex coupled atmosphere-ocean models.

\section{Acknowledgments}

This study was provided by the National Basic Research Program of China (Grant no. 2012CB417404), the National Nature Scientific Foundation of China (41230420), and the Basic Research Program of Qingdao Science and Technology Plan (11-1-4-95-jch).

\section{References}

[1] C. B. Vreugdenhil, Numerical Methods for Shallow Water Flow, Kluwer Academic Publishers, Boston, Mass, USA, 1994.

[2] S.-I. Iga and Y. Matsuda, "Shear instability in a shallow water model with implications for the Venus atmosphere," Journal of the Atmospheric Sciences, vol. 62, no. 7, part 2, pp. 2514-2527, 2005.

[3] J. Lambaerts, G. Lapeyre, and V. Zeitlin, "Moist versus dry barotropic instability in a shallow-water model of the atmosphere with moist convection," Journal of the Atmospheric Sciences, vol. 68, no. 6, pp. 1234-1252, 2011.

[4] S. Jiang, F. F. Jin, and M. Ghil, "Multiple equilibria, periodic and aperiodic solutions in a wind-driven double-gyre, shallowwater model," Journal of Physical Oceanography, vol. 25, pp. 764-786, 1995.

[5] F. Primeau, "Multiple equilibria and low-frequency variability of the wind-driven ocean circulation," Journal of Physical Oceanography, vol. 32, no. 8, pp. 2236-2256, 2002.

[6] E. Simonnet, M. Ghil, K. Ide, R. Temam, and S. Wang, "Lowfrequency variability in shallow-water models of the winddriven ocean circulation. I. Steady-state solution," Journal of Physical Oceanography, vol. 33, no. 4, pp. 712-728, 2003.

[7] F. W. Primeau and D. Newman, "Bifurcation structure of a wind-driven shallow water model with layer-outcropping," Ocean Modelling, vol. 16, no. 3-4, pp. 250-263, 2007.

[8] O. B. Andersen, "Shallow water tides in the northwest European shelf region from TOPEX/POSEIDON altimetry," Journal of Geophysical Research C: Oceans, vol. 104, no. 4, pp. 7729-7741, 1999.

[9] S. B. Yoon, C. H. Lim, and J. Choi, "Dispersion-correction finite difference model for simulation of transoceanic tsunamis," Terrestrial, Atmospheric and Oceanic Sciences, vol. 18, no. 1, pp. 31-53, 2007.

[10] N. V. Chemetov, F. Cipriano, and S. Gavrilyuk, "Shallow water model for lakes with friction and penetration," Mathematical Methods in the Applied Sciences, vol. 33, no. 6, pp. 687-703, 2010.

[11] J. Carlson, A. Jaffe, and A. Wiles, The Millennium Prize Problems, American Mathematical Society, 2006.

[12] S. Chippada, C. N. Dawson, M. L. Martinez, and M. F. Wheeler, "A Godunov-type finite volume method for the system of shallow water equations," Computer Methods in Applied Mechanics and Engineering, vol. 151, no. 1-2, pp. 105-129, 1998. 
[13] F. Alcrudo and P. Garcia-Navarro, "High-resolution Godunovtype scheme in finite volumes for the $2 \mathrm{D}$ shallow-water equations," International Journal for Numerical Methods in Fluids, vol. 16, no. 6, pp. 489-505, 1993.

[14] R. A. Walters, "Numerically induced oscillations in finite element approximations to shallow water equations," International Journal for Numerical Methods in Fluids, vol. 3, no. 6, pp. 591604, 1983.

[15] V. Casulli, "Semi-implicit finite difference methods for the twodimensional shallow water equations," Journal of Computational Physics, vol. 86, no. 1, pp. 56-74, 1990.

[16] G.-F. Lin, J.-S. Lai, and W.-D. Guo, "Finite-volume componentwise TVD schemes for 2D shallow water equations," Advances in Water Resources, vol. 26, no. 8, pp. 861-873, 2003.

[17] M. L. Batteen and Y. J. Han, "On the computational noise of finite-difference schemes used in ocean models," Svenska Geofysiska Föreningen, vol. 33, no. 4, pp. 387-396, 1981.

[18] V. Casullia and R. A. Waltersb, "An unstructured grid, threedimensional model based on the shallow water equations," International Journal for Numerical Methods in Fluids, vol. 32, pp. 331-348, 2000.

[19] W. C. Thacker, "Irregular grid finite-difference techniques: Simulations of oscillations in shallow circular basins," Journal of Physical Oceanography, vol. 7, no. 2, pp. 284-292, 1977.

[20] V. Casulli and R. T. Cheng, "Semi-implicit finite difference methods for three-dimensional shallow water flow," International Journal for Numerical Methods in Fluids, vol. 15, no. 6, pp. 629-648, 1992.

[21] Y. Xing and C.-W. Shu, "High order finite difference WENO schemes with the exact conservation property for the shallow water equations," Journal of Computational Physics, vol. 208, no. 1, pp. 206-227, 2005.

[22] R. D. Nair, S. J. Thomas, and R. D. Loft, "A discontinuous Galerkin global shallow water model," Monthly Weather Review, vol. 133, no. 4, pp. 876-888, 2005.

[23] G. Quattrocchi, S. Pierini, and H. A. Dijkstra, "Intrinsic lowfrequency variability of the Gulf Stream," Nonlinear Processes in Geophysics, vol. 19, no. 2, pp. 155-164, 2012.

[24] M. J. Schmeits and H. A. Dijkstra, "Bimodal behavior of the Kuroshio and the Gulf Stream," Journal of Physical Oceanography, vol. 31, no. 12, pp. 3435-3456, 2001.

[25] R. J. Greatbatch, X. Zhai, M. Claus, L. Czeschel, and W. Rath, "Transport driven by eddy momentum fluxes in the Gulf Stream Extension region," Geophysical Research Letters, vol. 37, no. 24, Article ID L24401, 2010.

[26] B. Qiu and W. Miao, "Kuroshio path variations south of Japan: bimodality as a self-sustained internal oscillation," Journal of Physical Oceanography, vol. 30, no. 8, pp. 2124-2137, 2000.

[27] Q. Wang, M. Mu, and H. A. Dijkstra, "Application of the conditional nonlinear optimal perturbation method to the predictability study of the Kuroshio large meander," Advances in Atmospheric Sciences, vol. 29, no. 1, pp. 118-134, 2012.

[28] S. Pierini, "A Kuroshio extension system model study: decadal chaotic self-Sustained oscillations," Journal of Physical Oceanography, vol. 36, no. 8, pp. 1605-1625, 2006.

[29] F. Primeau and D. Newman, "Elongation and contraction of the western boundary current extension in a shallow-water model: a bifurcation analysis," Journal of Physical Oceanography, vol. 38, no. 7, pp. 1469-1485, 2008.

[30] W. Kramer, H. A. Dijkstra, S. Pierini, and P. J. Van Leeuwen, "Measuring the impact of observations on the predictability of the kuroshio extension in a shallow-water model," Journal of Physical Oceanography, vol. 42, no. 1, pp. 3-17, 2012.

[31] G. M. Almeida, P. Bates, J. E. Freer, and M. Souvignet, "Improving the stability of a simple formulation of the shallow water equations for 2-D flood modeling," Water Resources Research, vol. 48, p. W05528, 2012.

[32] S. Sankaranarayanan and M. L. Spaulding, "Dispersion and stability analyses of the linearized two-dimensional shallow water equations in boundary-fitted co-ordinates," International Journal for Numerical Methods in Fluids, vol. 42, no. 7, pp. 741763, 2003.

[33] M. Israeli, N. H. Naik, and M. A. Cane, "An unconditionally stable scheme for the shallow water equations," Monthly Weather Review, vol. 128, no. 3, pp. 810-823, 2000.

[34] R. Heihes and D. A. Randall, "Numerical integration of the shallow-water equations on a twisted icosahedral grid-partI: basic design and results of tests," Monthly Weather Review, vol. 123, pp. 1862-1880, 1995.

[35] A. Grammeltvedt, "A survey of finite-difference schemes for the primitive equations for a barotropic fluid," Monthly Weather Review, vol. 97, pp. 384-404, 1969.

[36] T. C. Rebollo, A. D. Delgado, and E. D. Nieto, "A family of stable numerical solvers for the shallow water equations with source terms," Computer Methods in Applied Mechanics and Engineering, vol. 192, no. 1-2, pp. 203-225, 2003.

[37] J. D. Anderson, Computational Fluid Dynamics: The Basics with Application, McGraw-Hill Science, 1995.

[38] H. A. Dijkstra, Nonlinear Physical Oceanography: A Dynamical Systems Approach to Thelarge Scale Ocean Circulation and El Nino, Springer, Dordrecht, The Netherlands, 2nd edition, 2005.

[39] J. W. Thomas, Numerical Partial Differential Equations: Finite Difference Methods, vol. 22 of Texts in Applied Mathematics, Springer, New York, NY, USA, 1998.

[40] J. C. Strikwerda, Finite Difference Schemes and Partial Differential Equations, The Wadsworth \& Brooks/Cole Mathematics Series, Wadsworth \& Brooks, Pacific Grove, Calif, USA, 1989.

[41] B. Engquist and A. Majda, "Radiation boundary conditions for acoustic and elastic wave calculations," Communications on Pure and Applied Mathematics, vol. 32, no. 3, pp. 314-358, 1979.

[42] D. R. Durran, Numerical Methods for Fluid Dynamics, vol. 32 of Texts in Applied Mathematics, Springer, New York, NY, USA, 2nd edition, 2010.

[43] A. F. Blumberg and G. L. Mellor, "A description of a threedimensional coastal ocean model," in Three-Dimensional Coastal Ocean Models, Coastal and Estuarine Sciences, vol. 32, pp. 1$16,1987$. 


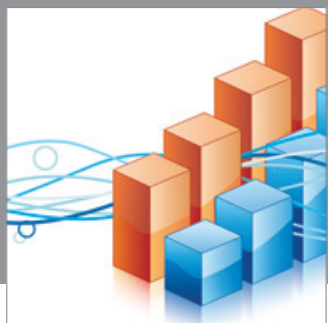

Advances in

Operations Research

mansans

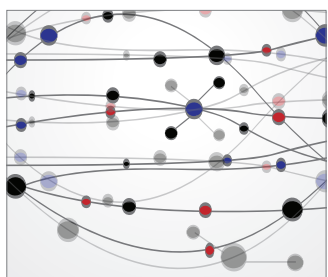

The Scientific World Journal
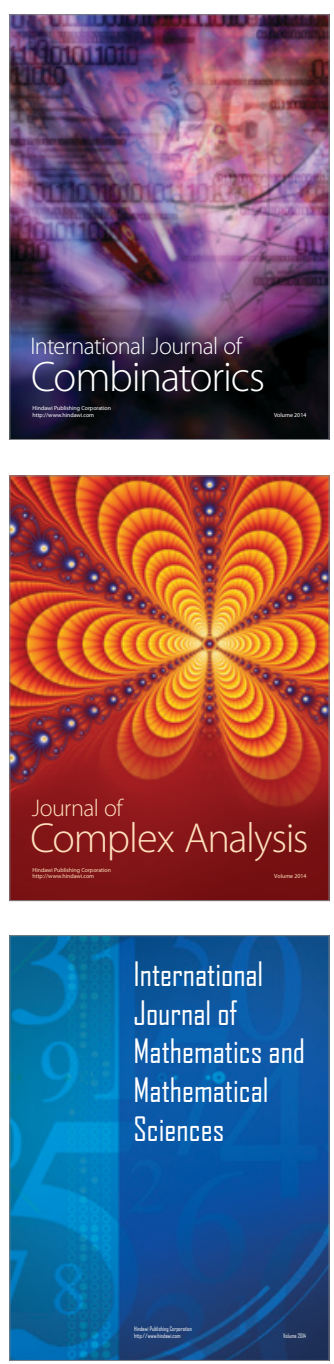
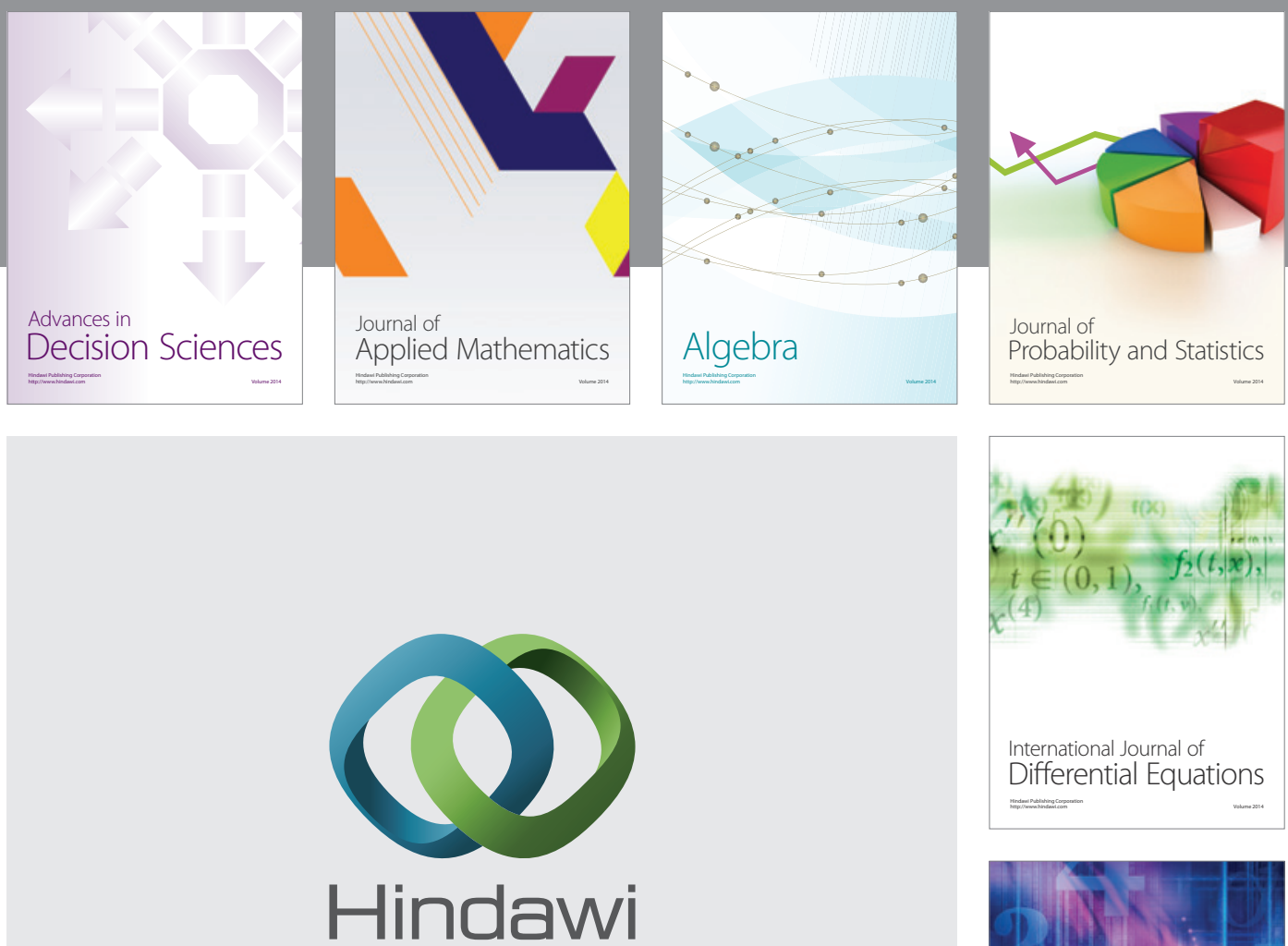

Submit your manuscripts at http://www.hindawi.com
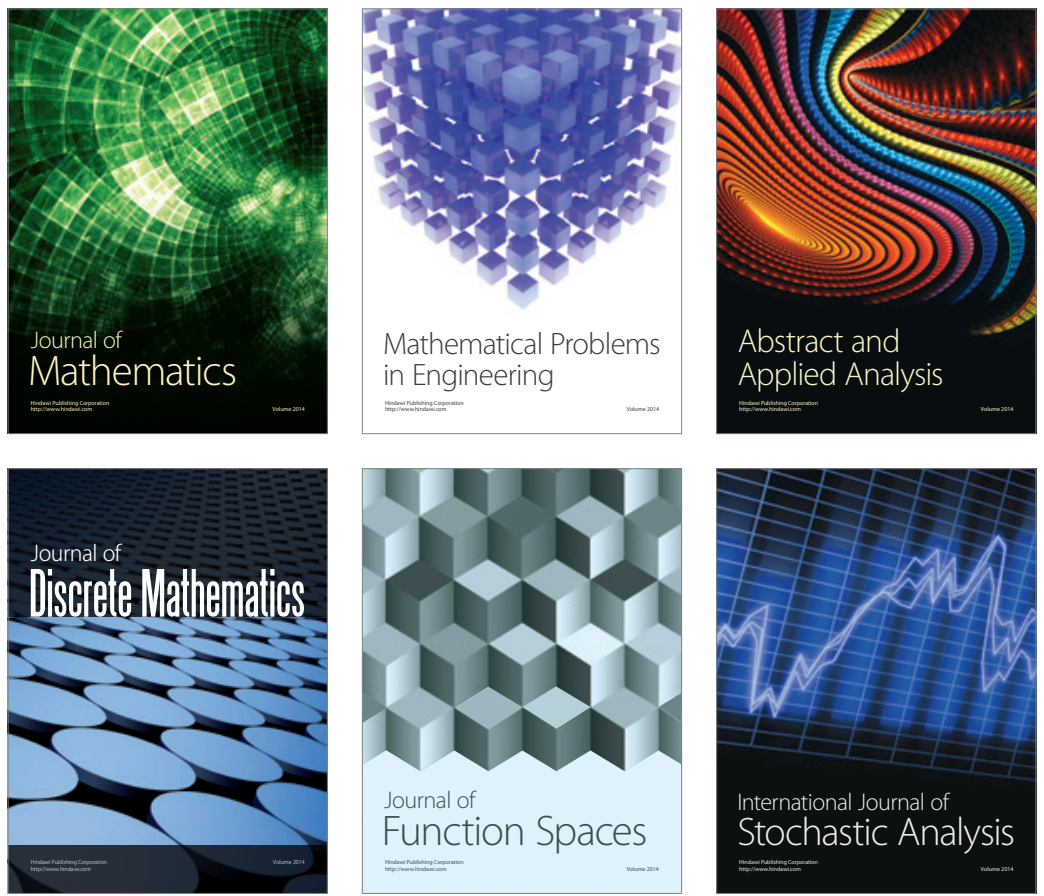

Journal of

Function Spaces

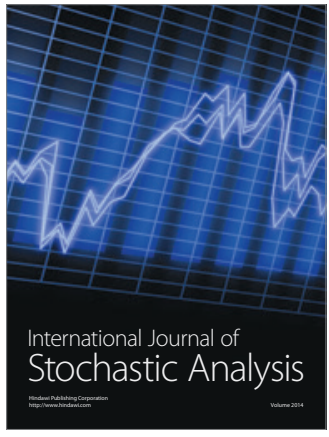

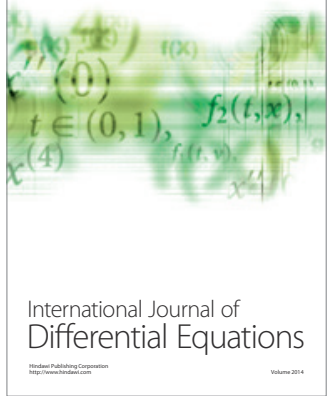
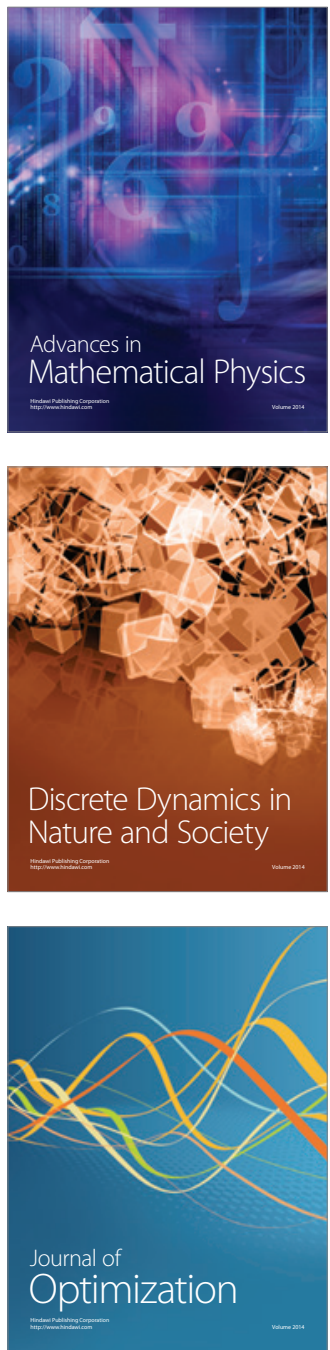